\title{
Accessibility, reliability, and usability of neurosurgical resources
}

\author{
Nitin Agarwal, MD, ${ }^{1}$ Sumana S. Kommana, BS, ${ }^{2}$ David R. Hansberry, MD, PhD, ${ }^{3}$ \\ Ahmed I. Kashkoush, BS, ${ }^{1}$ Robert M. Friedlander, MD, MA, ${ }^{1}$ and L. Dade Lunsford, MD1 \\ 1Department of Neurological Surgery, University of Pittsburgh Medical Center, Pittsburgh, Pennsylvania; ${ }^{2}$ Rutgers New Jersey \\ Medical School, Newark, New Jersey; and ${ }^{3}$ Department of Radiology, Thomas Jefferson University Hospitals, Philadelphia, \\ Pennsylvania
}

OBJECTIVE Closing the knowledge gap that exists between patients and health care providers is essential and is facilitated by easy access to patient education materials. Although such information has the potential to be an effective resource, it must be written in a user-friendly and understandable manner, especially when such material pertains to specialized and highly technical fields such as neurological surgery. The authors evaluated the accessibility, usability, and reliability of current educational resources provided by the American Association of Neurological Surgeons (AANS), Healthwise, and the National Institute for Neurological Disorders and Stroke (NINDS).

METHODS Online neurosurgical patient education information provided by AANS, Healthwise, and NINDS was evaluated using the LIDA scale, a website quality assessment tool, by medical professionals and nonmedical professionals. A high achieving score is regarded as $90 \%$ or greater using the LIDA scale.

RESULTS Accessibility scores were 76.7\% (AANS), 83.3\% (Healthwise), and 75.0\% (NINDS). Average usability scores for the AANS, Healthwise, and NINDS were $73.3 \%, 82.6 \%$, and $82.9 \%$, respectively, when evaluated by medical professionals and $78.5 \%, 80.7 \%$, and $75.9 \%$, respectively, for nonmedical professionals, respectively. Average reliability scores were $58.5 \%, 53.3 \%, 72.6 \%$, respectively, for medical professionals and $70.4 \%, 66.7 \%$, and $78.5 \%$, respectively, for nonmedical professionals when evaluating the AANS, Healthwise, and NINDS websites.

CONCLUSIONS Although organizations like AANS, Healthwise, and NINDS should be commended for their ongoing commitment to provide health care-oriented materials, modification of this material is suggested to improve the patient education value.

https://thejns.org/doi/abs/10.3171/2015.12.JNS151102

KEY WORDS accessibility; reliability; usability; patient education

$\mathrm{T}$ HE value of patient education resources has grown over the past decade with the increased use of the Internet, which has become a widely available method to inform patients, health care providers, and the general public. ${ }^{31}$ Medical terminology and discussion of management options can often be cryptic and intimidating to nonmedical persons, who frequently access the Internet for answers to their questions. In its 2013 study, the Pew
Internet and American Life Project reported that $81 \%$ of US adults use the Internet and 59\% searched online for health care information in the past year. Additionally, 35\% of US adults access the Internet specifically to learn about a particular medical condition or an unfamiliar drug. ${ }^{20}$ With the increased availability of online health care resources within the Internet's vast reserve of information, material that was once difficult to access is now only a click away. ${ }^{16}$

ABBREVIATIONS AANS = American Association of Neurological Surgeons; FRE = Flesch Reading Ease; HON = Health on the Net; NINDS = National Institute for Neurological Disorders and Stroke; PEMAT = Patient Education Materials Assessment Tool; QUIS = Questionnaire for User Interaction Satisfaction; SUS = Systems Usability Scale; UPMC = University of Pittsburgh Medical Center.

SUBMITTED May 11, 2015. ACCEPTED December 29, 2015.

INCLUDE WHEN CITING Published online June 3, 2016; DOI: 10.3171/2015.12.JNS151102. 
As an increasing number of professional organizations offer website sections devoted to patient education, there is increasing apprehension that some information may be either incorrect or inaccurate. ${ }^{8}$ The Internet has the potential to eliminate barriers to accessing information for patients, but only if online material can be read and understood by users with various levels of medical sophistication. ${ }^{16,21}$ When assessing the quality of information from a certain source, availability and accuracy are two factors that are frequently used.

Previous studies have focused on evaluating the readability of patient education resources. ${ }^{1-4,23-29}$ Understanding and proper utilization of these resources may require a level beyond the recommended fourth to sixth grade reading level. ${ }^{3,4}$ This would leave patients at lower reading levels at a disadvantage..$^{14,17-19}$ However, a variety of instruments have been developed to further evaluate the quality of the information on the Internet. One such tool is the LIDA instrument that analyzes the accessibility, usability, and reliability of a website. ${ }^{11}$

The aim for any online resource is to be accessible, comprehensible, navigable, dependable, and unbiased..$^{33}$ The LIDA scale, a validation instrument for health care websites, is a 41-question instrument used to provide an assessment of any health care website (http://www.miner vation.com/wp-content/uploads/2011/04/MinervationLIDA-instrument-v1-2.pdf). Since the tool's development in 2007, at least 13 publications have used it (along with other tools) to evaluate a variety of health care websites. ${ }^{15}$ Furthermore, the creators of the LIDA scale have provided some formal testing on the tool's internal validity. Forty major websites about prostate cancer were selected and analyzed utilizing the LIDA scale. The correlation between each grader's rank order was noted to be statistically significant $(\mathrm{p}<0.00001){ }^{32}$ To date, the LIDA tool has not been formally compared with other routinely used measures of health website quality, such as the Flesch Reading Ease (FRE) score, and thus it is difficult to judge the external validity of the quality measurement. However, multiple studies have demonstrated a correlation between LIDA and FRE scores. ${ }^{5,22}$ Previous studies have demonstrated that the FRE scores for neurosurgical patient education resources have been low, corresponding to increased difficulty of reading and perhaps lower quality. ${ }^{1,2,4}$ This study evaluates the websites of the American Association of Neurological Surgeons (AANS), the University of Pittsburgh Medical Center (UPMC; Healthwise Inc.), and the National Institute of Neurological Disorders and Stroke (NINDS), focusing on the quality of patient education materials provided with regards to their accessibility, usability, and reliability.

\section{Methods \\ LIDA Scale}

The AANS website provides patient education articles specifically written for the public (http://www.aans.org/). These articles were subsequently compared for their level of accessibility, usability, and reliability using the LIDA scale. This process was also performed for patient education materials from UPMC (http://www.upmc.com/Pages/ default.aspx), which are provided by Healthwise Incorporated, and NINDS (http://www.ninds.nih.gov/). The LIDA instrument automatically provides results for accessibility and obtains results for utility and reliability using a series of questions that an evaluator answers. Each category is scored on a scale of $0-3$, where $0=$ never, $1=$ sometimes, $2=$ mostly, $3=$ always. LIDA scores were ranked as high if they were $>90 \%$, moderate if they were between $50 \%$ and $90 \%$, and low if they were $<50 \% .^{11}$

Accessibility is defined by a set of legal guidelines provided by the World Wide Web Consortium on requirements and suggestions for how to maximize accessibility of a web page to provide those with disabilities an equal opportunity to use the Internet. ${ }^{13}$ Full text should be able to be accessed without registration or a fee and the web page should be compatible with all commonly used web browsers, such as Firefox and Google Chrome. The information should also be available without outdated HTML codes. This information was automatically assessed by the online LIDA assessment tool (http://www.minervation. com/lida-tool/). The maximum possible score was 60 .

Usability of the website evaluates how easy the website is to follow and whether users can find the information they need. Criteria assessed in this category include clarity, consistency, functionality, and the ability to engage. The maximum possible score was 54 .

The LIDA instrument also measures the reliability of patient education resources. The measures assessed included currency of the material, conflicts of interest, and content production. The maximum possible score was 27 .

Utilizing the LIDA scale, the AANS website was evaluated independently by 5 independent medical professionals and 5 nonmedical professionals from various areas of the country in September 2015. Subjects were included in the medical professional group if they had received any medical training, and included physicians and medical students. Nonmedical professionals included people who did not have a medical degree, were not in medical school, and did not work in the health care industry. One-way ANOVA testing was used to assess for similarity between AANS, Healthwise, and NINDS usability and reliability scores. Usability and reliability scores were averaged, and a 2-tailed Student t-test was used for comparison of the mean scores between the 2 cohorts of subjects. Accessibility scores were generated automatically by the LIDA tool with no input from subjects and thus were not subjected to statistical analysis.

\section{Results}

The LIDA scale determined that the AANS, Healthwise, and NINDS websites had accessibility, usability, and reliability scores classified as moderate $(50 \%-90 \%)$ when assessed by both medically and nonmedically oriented subjects (Tables 1 and 2).

\section{Accessibility}

With regards to accessibility, the AANS (76.7\%), Healthwise (83.3\%), and NINDS (75.0\%) websites were moderate as scored with the LIDA scale. The LIDA instrument automatically scored key points, such as validity 
TABLE 1. LIDA scores of usability and reliability as administered by medical professionals

\begin{tabular}{lrrrrrrr}
\hline & \multicolumn{9}{c}{ Rater } & $\begin{array}{c}\text { Average } \\
\text { Score (\%) }\end{array}$ & $\begin{array}{c}95 \% \\
\text { Wl }\end{array}$ \\
\cline { 2 - 7 } & 1 & 2 & 3 & 4 & 5 & & \\
\hline Usability* & & & & & & & \\
\hline AANS & 43 & 42 & 35 & 43 & 35 & $39.6(73.3)$ & $34.4-44.8$ \\
\hline Healthwise & 49 & 47 & 46 & 38 & 43 & $44.6(82.6)$ & $39.3-49.9$ \\
\hline NINDS & 48 & 45 & 48 & 38 & 45 & $44.8(82.9)$ & $39.7-49.9$ \\
\hline Total & & & & & & $43.0(79.6)$ & $40.4-45.6$ \\
\hline Reliability $\dagger$ & & & & & & & \\
\hline AANS & 19 & 19 & 19 & 11 & 11 & $15.8(58.5)$ & $10.4-21.2$ \\
\hline Healthwise & 14 & 21 & 21 & 6 & 10 & $14.4(53.3)$ & $6.1-22.7$ \\
\hline NINDS & 16 & 25 & 26 & 15 & 16 & $19.6(72.6)$ & $12.9-26.3$ \\
\hline Total & & & & & & $16.6(61.5)$ & $13.5-19.7$ \\
\hline
\end{tabular}

${ }^{*}$ Maximum score $=54$.

$\dagger$ Maximum score $=27$.

of the HTML code, access restrictions (including for those with disabilities), and web page setup, which encompasses the presence of a page title as well as optimal alignment of text and images. However, subject input was required for access level and browser compatibility. All 3 websites do not have a registration requirement or fees for access, which greatly alleviates some of the accessibility burdens for users.

\section{Usability}

With regards to usability, all 3 websites were scored as moderate across both cohorts (Tables 1 and 2). Medical professionals gave scores of $73.3 \%, 82.6 \%$, and $82.9 \%$, whereas nonmedical professionals reported scores of $78.5 \%, 80.7 \%$, and $75.9 \%$ for the AANS, Healthwise, and NINDS websites, respectively. No statistically significant differences were observed across the 3 websites via 1-way ANOVA testing in both cohorts (professionals, $\mathrm{p}=0.127$; nonmedical professionals, $\mathrm{p}=0.600$ ). A 2-tailed Student t-test was performed to compare usability scores between the 2 cohorts, and no statistically significant differences were found ( $\mathrm{p}=0.671$; Table 3 ).

Subjects were then asked to provide overall feedback about the 3 websites. Given the large amounts of information initially presented to users of the AANS website, users recommended highlighting the patient education access tab, because the navigation bar at the top was crowded. Subjects felt that the color template used on the website had visual appeal, even for long periods of time. However, the article titles in the initial list of topics were written in a faint green color and thus could be difficult for some to read. Users also noted the lack of graphics and other nontextual media covering patient education materials across all 3 websites. Moreover, readers did not have the ability to differentiate between previously clicked links on the AANS and UPMC websites.

Utilizing the general search box, users observed that an overwhelming number of results were queried. For example, after "brain tumor" is entered, the AANS site yields over 1400 results. However, the website does have an ad-
TABLE 2. LIDA scores of usability and reliability as administered by nonmedical professionals

\begin{tabular}{lccccccc}
\hline & \multicolumn{9}{c}{ Rater } & $\begin{array}{c}\text { Average } \\
\text { Website }\end{array}$ & 1 & 2 & 3 & 4 & 5 & Score $(\%)$ & $95 \% \mathrm{Cl}$ \\
\hline Usability & & & & & & & \\
\hline AANS & 41 & 41 & 41 & 48 & 41 & $42.4(78.5)$ & $38.5-46.3$ \\
\hline Healthwise & 44 & 43 & 41 & 46 & 44 & $43.6(80.7)$ & $41.3-45.9$ \\
\hline NINDS & 35 & 42 & 45 & 48 & 35 & $41.0(75.9)$ & $33.7-48.3$ \\
\hline Total & & & & & & $42.3(78.3)$ & $40.2-44.5$ \\
\hline Reliability $\dagger$ & & & & & & & \\
\hline AANS & 17 & 16 & 19 & 26 & 17 & $19.0(70.4)$ & $14.0-24.0$ \\
\hline$\quad$ Healthwise & 18 & 17 & 14 & 23 & 18 & $18.0(66.7)$ & $14.0-22.0$ \\
\hline$\quad$ NINDS & 22 & 17 & 20 & 25 & 22 & $21.2(78.5)$ & $17.5-24.9$ \\
\hline Total & & & & & & $19.4(71.9)$ & $17.5-21.3$ \\
\hline * Maximum score $=54$. & & & & & \\
$\dagger$ Maximum score $=27$. & & & & &
\end{tabular}

vanced search option where readers are allowed to refine their search based on category, folder, and date of release. This presents the reader with a much more manageable list of related hits that the reader can search for the desired information.

\section{Reliability}

With regards to reliability, all 3 websites were scored as moderate with the LIDA scale by both cohorts (Tables 1 and 2). Medical professionals gave scores of 58.5\%, 53.3\%, and $72.6 \%$, whereas nonmedical professionals reported scores of $70.4 \%, 66.7 \%$, and $78.5 \%$ for AANS, Healthwise, and NINDS websites, respectively. No statistically significant differences were observed for reliability across the 3 websites when analyzed with 1-way ANOVA testing in both cohorts (professionals, $\mathrm{p}=0.343$; nonmedical professionals, $p=0.356$ ). Furthermore, a 2-tailed Student t-test was used to compare reliability scores between the 2 cohorts, and no statistically significant differences were found ( $\mathrm{p}=0.115$; Table 3 ).

Reliability was evaluated based on the coverage of recent events on the website as well as the presence of a user comment section. There is a clear section on the AANS page dedicated to recent events on the home page that is even further subdivided into patient news, AANS news, and general clinical news. While this helped increase its reliability score, the lack of a user feedback section detracted

TABLE 3. Two-tailed Student t-test for comparison between both cohorts*

\begin{tabular}{lccc}
\hline Parameter & $\begin{array}{c}\text { Medical Professional } \\
\text { Scores (\%) }\end{array}$ & $\begin{array}{c}\text { Nonprofessional } \\
\text { Scores (\%) }\end{array}$ & p Value \\
\hline Usability & $43.0(79.6)$ & $42.3(78.3)$ & 0.671 \\
\hline Reliability & $16.6(61.5)$ & $19.4(71.9)$ & 0.115 \\
\hline * Cohorts included scores for 5 medical and 5 nonmedical professionals at 3 \\
$\begin{array}{l}\text { websites; therefore, each cohort contained } 15 \text { scores. The percentages are } \\
\text { derived by taking the score and dividing it by the maximum possible score for } \\
\text { each test. }\end{array}$
\end{tabular}


from the score. Additionally, users generally appreciated that all 3 websites had links for additional resources. For instance, the NINDS page on "Back Pain" provides links to the American Chronic Pain Association as well as a "Low Back Pain Fact Sheet," among others. However, both cohorts of subjects criticized the lack of citations and references for the material and facts provided across all 3 websites. None of the articles cite references, which reduces the reliability of the information provided because the information cannot be checked against the original sources.

\section{Discussion}

Specific surgical subspecialties, such as neurological surgery, are fields that patients typically have limited personal experience with. By reviewing information on specific conditions, a patient is able to quickly access a wealth of information that will provide the dual benefit of assuaging their fears while also providing them context. In turn, this may lead to increased patient satisfaction and improved access to various care options.

The Internet has become the single largest and most extensively used information source. Up to $80 \%$ of the population uses the Internet regularly and more than half the population with Internet access seek information related to health care at least once a month., ${ }^{9,20}$ Although free access to online material has empowered the public with the ability to obtain a great amount of medical information, there are few restrictions and guidelines overseeing the quality of the information..$^{10}$ Users searching for health care information online could be at a high risk of finding unreliable information as well as information that is inadequate and inappropriate regarding the topic of interest. ${ }^{12}$ As such, lower-quality resources may negatively influence health care-related decisions. Thus, the issue is not one of quantity but of quality. ${ }^{11}$

\section{Alternative Methods of Quality Assessment}

Accessibility, usability, and reliability are 3 domains of interest that have been routinely used to measure the quality of a health website. Examples of other scales evaluating these domains include the Systems Usability Scale (SUS), ${ }^{6}$ the Questionnaire for User Interaction Satisfaction (QUIS), ${ }^{30}$ and DISCERN, an instrument to help consumers judge the quality of available health information about treatment choices. ${ }^{6,7}$ The SUS and DISCERN questionnaires can be used to assess usability, while the QUIS is a 27-question survey that may be used to evaluate reliability. However, the LIDA scale offers a potential evaluator the ability to the measure accessibility, usability, and reliability, and, as such, was chosen as the primary assessment tool for this study.

Additionally, the Patient Education Materials Assessment Tool (PEMAT), developed by the Agency for Healthcare Research and Quality, is another systematic method to evaluate and compare the "understandability" and "actionability" of patient education materials. The PEMAT may be used only for audiovisual or printable materials. While the PEMAT cannot be used to evaluate podcasts or the usability of websites, it may be a very valuable tool when used in conjunction with the LIDA tool.

\section{Future Directions and Limitations}

Currently, most medical societies have their own website with a section dedicated to patient education. However, it may be beneficial to create one uniform method to present patient education resources that is accessible, useful, and reliable. Herein, the different authors and societies could be mandated to follow a structured format or be overseen by a single authority that helps to maintain consistency across the materials. Additionally, this information may be further validated (for example) by the Health on the Net (HON) code, which is a certification system of 8 standards governing authorship, complementarity, confidentiality, attribution, justifiability, transparency, financial disclosure, and advertising. The HON code was created by the HON Foundation, a nongovernmental organization recognized for its work in the field of health information ethics.

In terms of the overall appearance of the neurosurgical websites, they are presented in a relatively organized and appealing manner but a few areas have the potential for improvement. The articles, in addition to text, could be enriched with other types of learning media, such as images and videos. These, combined with the text, could act as supplements to the more difficult topics covered on the site. A benefit of using multimedia instruction, such as video, is the increased ability of the user to understand and retain the material. ${ }^{34}$ Moreover, for those who struggle with reading, video tutorials would offer an alternative method to receive the same information. Additional features for patients with visual disabilities are suggested. For instance, frequently websites have larger font options to aid those with impaired vision and black and white formatting for the colorblind. This point is emphasized with respect to the light green color scheme used for the hyperlinks on the AANS website. Moreover, it may be beneficial to have links change to another color after being visited, so the reader can keep track of what has been read and what is yet to be explored.

With regard to reliability, the use of regular citations throughout patient education materials should be considered. This was a common criticism across both cohorts of subjects. Directing a medically illiterate audience to the primary research may seem paradoxical due to the complexity of medical literature. However, the added benefit of listing the original sources is increased transparency of the website's content production, which increases the information's reputability. Citing the primary research further empowers patients, because it provides avenues for credible and factual medical information, which they can discuss in the appropriate amount of detail with their physician. By withholding citations to credible sources, the medical community only limits patients and leaves them vulnerable to unreliable and nonfactual sources of information, which can impair their decision making. Providing original literature may also benefit patients with a higher level of education who may be able to understand complex information but simply lack the insight or patience to conduct a focused literature review.

Of interest is the lack of significant differences in reliability scores between the 2 cohorts of subjects (Table 3 ). One might assume that medical professionals would find the resources in this study less reliable than nonmedical professionals would due to the overly simplistic language used throughout. Similarity between the scores of medical 
professionals and nonmedical professionals could be a consequence of the LIDA survey's robustness and objectivity. However, this study ultimately has limitations, with the most evident being the modest sample size of evaluators. Having more individuals analyze the website would increase the statistical significance of our results and limit bias that might influence the individual analysis. Still, the potential website revisions advocated for by the study have the potential to greatly improve a user's experience and access to the public education material.

\section{Conclusions}

With the continual evolution of the Internet, guidelines and regulation of the information must be set to prevent the distribution of false information that can potentially do harm to the reader. The Plain Writing Act of 2010 requires that federal agencies use "clear Government communication that the public can understand and use." In conjunction, the Plain Language Action and Information Network serves as a community of federal employees dedicated to the idea that citizens deserve clear communications from government. Similar guidelines and agencies need to be developed and applied specifically for the field of health care. Many health care-related answers are sought out via the Internet to alleviate some of the anxiety and curiosity that patients may have before or after meeting with their physician. With this analysis, it is clear that the neurosurgical websites in this study provide information of a high level of quality that is accessible, usable, and reliable; however, there are areas that if improved would likely further benefit those who visit the websites. Further evaluation with additional user feedback is recommended.

\section{References}

1. Agarwal N, Chaudhari A, Hansberry DR, Tomei KL, Prestigiacomo CJ: A comparative analysis of neurosurgical online education materials to assess patient comprehension. J Clin Neurosci 20:1357-1361, 2013

2. Agarwal N, Feghhi DP, Gupta R, Hansberry DR, Quinn JC, Heary RF, et al: A comparative analysis of minimally invasive and open spine surgery patient education resources. J Neurosurg Spine 21:468-474, 2014

3. Agarwal N, Hansberry DR, Sabourin V, Tomei KL, Prestigiacomo CJ: A comparative analysis of the quality of patient education materials from medical specialties. JAMA Intern Med 173:1257-1259, 2013

4. Agarwal N, Sarris C, Hansberry DR, Lin MJ, Barrese JC, Prestigiacomo CJ: Quality of patient education materials for rehabilitation after neurological surgery. NeuroRehabilitation 32:817-821, 2013

5. Alsafi A, Kaya G, Patel H, Hamady MS: A comparison of the quality of the information available on the internet on interventional radiology, vascular surgery, and cardiology. J Postgrad Med 59:69-75, 2013

6. Brooke J: SUS: a 'quick and dirty' usability scale, in Jordan PW, Thomas B McClelland IL, et al (eds): Usability Evaluation in Industry. London: Taylor \& Francis, 1996

7. Charnock D: The Discern Handbook. Quality Criteria for Consumer Health Information on Treatment Choices. Abingdon, UK: Radcliffe, 1998

8. Coleman M, Liau TL: A computer readability formula designed for machine scoring. J Appl Psychol 60:283-284, 1975
9. Cotugna N, Vickery CE, Carpenter-Haefele KM: Evaluation of literacy level of patient education pages in health-related journals. J Community Health 30:213-219, 2005

10. D'Alessandro DM, Kingsley P, Johnson-West J: The readability of pediatric patient education materials on the World Wide Web. Arch Pediatr Adolesc Med 155:807-812, 2001

11. Davis TC, Williams MV, Marin E, Parker RM, Glass J: Health literacy and cancer communication. CA Cancer J Clin 52:134-149, 2002

12. Diaz JAGR, Griffith RA, Ng JJ, Reinert SE, Friedmann PD, Moulton AW: Patients' use of the Internet for medical information. J Gen Intern Med 17:180-185, 2002

13. Dollahite J, Thompson C, McNew R: Readability of printed sources of diet and health information. Patient Educ Couns 27:123-134, 1996

14. Eysenbach G: The impact of the Internet on cancer outcomes. CA Cancer J Clin 53:356-371, 2003

15. Feghhi DP, Komlos D, Agarwal N, Sabharwal S: Quality of online pediatric orthopaedic education materials. J Bone Joint Surg Am 96:e194, 2014

16. Ferguson GG, Eliasziw M, Barr HW, Clagett GP, Barnes RW, Wallace MC, et al: The North American Symptomatic Carotid Endarterectomy Trial: surgical results in 1415 patients. Stroke 30:1751-1758, 1999

17. Flesch RF: How to Write Plain English: A Book for Lawyers and Consumers, ed 1. New York: Harper \& Row, 1979

18. Fox S: Mobile health 2010. Pew Research Center.. (http:// www.pewinternet.org/2010/10/19/mobile-health-2010/) [Accessed February 25, 2016]

19. Fox S: The social life of health information, 2011. Pew Research Center. (http://www.pewinternet.org/2011/05/12/ the-social-life-of-health-information-2011/) [Accessed February 25, 2016]

20. Fox S, Duggan M: Health online 2013. Pew Research Center. (http://www.pewinternet.org/2013/01/15/healthonline-2013/) [Accessed February 25, 2016]

21. Friedman DB, Hoffman-Goetz L: A systematic review of readability and comprehension instruments used for print and web-based cancer information. Health Educ Behav 33:352-373, 2006

22. Grewal P, Williams B, Alagaratnam S, Neffendorf J, Soobrah R: Quality of vascular surgery Web sites on the Internet. J Vasc Surg 56:1461-1467, 2012

23. Hansberry DR, Agarwal N, Gonzales SF, Baker SR: Are we effectively informing patients? A quantitative analysis of online patient education resources from the American Society of Neuroradiology. AJNR Am J Neuroradiol 35:1270-1275, 2014

24. Hansberry DR, John A, John E, Agarwal N, Gonzales SF, Baker SR: A critical review of the readability of online patient education resources from RadiologyInfo.org. AJR Am J Roentgenol 202:566-575, 2014

25. Hansberry DR, Kraus C, Agarwal N, Baker SR, Gonzales SF: Health literacy in vascular and interventional radiology: a comparative analysis of online patient education resources Cardiovasc Intervent Radiol 37:1034-1040, 2014

26. Hansberry DR, Agarwal N, Baker SR: Health literacy and online educational resources: an opportunity to educate patients. AJR Am J Roentgenol 204:111-116, 2015

27. Hansberry DR, Agarwal N, Shah R, Schmitt PJ, Baredes S, Setzen M, et al: Analysis of the readability of patient education materials from surgical subspecialties. Laryngoscope 124:405-412, 2014

28. Hansberry DR, Ramchand T, Patel S, Kraus C, Jung J, Agarwal N, et al: Are we failing to communicate? Internetbased patient education materials and radiation safety. Eur J Radiol 83:1698-1702, 2014 
29. Hansberry DR, Suresh R, Agarwal N, Heary RF, Goldstein IM: Quality assessment of online patient education resources for peripheral neuropathy. J Peripher Nerv Syst 18:44-47, 2013

30. Harper BD, Norman KL: Improving user satisfaction: The questionnaire for user interaction satisfaction version 5.5, (http://www.lap.umd.edu/QUIS/publications/harper1993.pdf) [Accessed February 25, 2016]

31. Maximus: Health Literacy Style Manual. Reston, VA: Maximus, 2005 (http://www.coveringkidsandfamilies.org/ resources/docs/stylemanual.pdf ) [Accessed February 25, 2016]

32. Minervation: Is the Lida website assessment tool valid? (http://www.minervation.com/does-lida-work/) [Accessed February 25, 2016]

33. Roshan A, Agarwal S, England RJ: Role of information available over the internet: what are the parents of children undergoing tonsillectomy likely to find? Ann R Coll Surg Engl 90:601-605, 2008

34. Stegeman CA, Zydney J: Effectiveness of multimedia instruction in health professions education compared to traditional instruction. J Dent Hyg 84:130-136, 2010

\section{Disclosures}

Dr. Lundsford is a consultant for AB Elekta and Insightec DSMB and also owns AB Elekta stock.

\section{Author Contributions}

Conception and design: Agarwal. Acquisition of data: Agarwal, Kommana. Analysis and interpretation of data: Agarwal, Kommana, Hansberry. Drafting the article: Agarwal, Kommana, Lunsford. Critically revising the article: all authors. Reviewed submitted version of manuscript: all authors. Approved the final version of the manuscript on behalf of all authors: Agarwal. Statistical analysis: Hansberry, Kashkoush. Administrative/ technical/material support: Friedlander, Lunsford. Study supervision: Lunsford.

\section{Correspondence}

Nitin Agarwal, Department of Neurological Surgery, University of Pittsburgh Medical Center, 200 Lothrop St., Ste. B-400, Pittsburgh, PA 15213-2582. email: agarwaln@upmc.edu. 